\section{Comprehensive analysis of dose intensity of acute lymphoblastic leukemia chemotherapy}

Seth E. Karol, ${ }^{1,2}$ Deqing Pei, ${ }^{3}$ Colton A. Smith, ${ }^{1}$ Yiwei Liu, ${ }^{1}$ Wenjian Yang, ${ }^{1}$ Nancy M. Kornegay, ${ }^{1}$ John C. Panetta, ${ }^{1}$ Kristine R. Crews, ${ }^{1}$ Cheng Cheng, ${ }^{3}$ Emily R. Finch, ${ }^{1}$ Hiroto Inaba, ${ }^{2}$ Monika L. Metzger, ${ }^{2,4}$ Jeffrey E. Rubnitz, ${ }^{2}$ Raul C. Ribeiro, ${ }^{2}$ Tanja A. Gruber, ${ }^{5,6}$ Jun J. Yang, ${ }^{1}$ William E. Evans, ${ }^{1}$ Sima Jeha, ${ }^{2,4}$ Ching-Hon Pui ${ }^{2}$ and Mary V. Relling ${ }^{1}$

${ }^{1}$ Department of Pharmaceutical Sciences, St. Jude Children's Research Hospital, Memphis, TN; 'Department of Oncology, St. Jude Children's Research Hospital, Memphis, TN; ${ }^{3}$ Department of Biostatistics, St. Jude Children's Research Hospital, Memphis, TN; ${ }^{4}$ Department of Global Pediatric Medicine, St. Jude Children's Research Hospital, Memphis, TN; ${ }^{5}$ Department of Pediatrics, Stanford University School of Medicine, Stanford, CA, and ${ }^{6}$ Stanford Cancer Institute, Stanford University School of Medicine, Stanford, CA, USA

\section{ABSTRACT}

$\longrightarrow$ hemotherapy dosages are often compromised, but most reports lack data on dosages that are actually delivered. In two consecutive acute lymphoblastic leukemia trials that differed in their asparaginase formulation, native E. coli L-asparaginase in St. Jude Total 15 (T15, $\mathrm{n}=365)$ and pegaspargase in Total $16(\mathrm{~T} 16, \mathrm{n}=524)$, we tallied the dose intensities for all drugs on the low-risk or standard-risk arms, analyzing 504,039 dosing records. The median dose intensity for each drug ranged from $61-100 \%$. Dose intensities for several drugs were more than $10 \%$ higher on T15 than on T16: cyclophosphamide $(P<0.0001$ for the standard-risk arm), cytarabine ( $P<0.0001$ for the standard-risk arm), and mercaptopurine $(P<0.0001$ for the low-risk arm and $P<0.0001$ for the standardrisk arm). We attributed the lower dosages on T16 to the higher asparaginase dosages on T16 than on T15 $(P<0.0001$ for both the low-risk and standard-risk arms), with higher dose-intensity for mercaptopurine in those with anti-asparaginase antibodies than in those without $(P=5.62 \times 10$ ${ }^{3}$ for T15 standard risk and $P=1.43 \times 10^{-4}$ for T16 standard risk). Neutrophil count did not differ between protocols for low-risk patients $(P=0.18)$ and was actually lower for standard-risk patients on T16 than on T15 $(P<0.0001)$ despite lower dosages of most drugs on T16. Patients with low asparaginase dose intensity had higher methotrexate dose intensity with no impact on prognosis. The only dose intensity measure predicting a higher risk of relapse on both studies was higher mercaptopurine dose intensity, but this did not reach statistical significance $(P=0.03$ T15; $P=0.07$ T16). In these intensive multiagent trials, higher dosages of asparaginase compromised the dosing of other drugs for acute lymphoblastic leukemia, particularly mercaptopurine, but lower chemotherapy dose intensity was not associated with relapse.

\section{Introduction}

Dosages of chemotherapy drugs used in treatment regimens for acute lymphoblastic leukemia (ALL) vary widely. ${ }^{1.8}$ There is, however, a lack of data comparing administered dosages to planned dosages, and the last comprehensive analysis (for only 209 patients) was published in $1991 .{ }^{9}$ Hence, it is difficult to compare feasibility of protocol delivery among cooperative treatment groups, between adults and children, and across different countries. Most ALL drugs can cause myelosuppression, and thus comparisons of administered dose intensity across protocols that are limited to a single agent, e.g., mercaptopurine or asparaginase, may be misleading if the dosages of other possibly "compensating" agents are not also accounted for across protocols. As newer immune-based and less myelosuppressive agents are added to
Ferrata Storti Foundation
Haematologica 2022

Volume 107(2):371-380

\section{Correspondence: \\ MARY V. RELLING \\ mary.relling@stjude.org}

Received: January 21, 2021.

Accepted: April 28, 2021.

Pre-published: July 1, 2021.

https://doi.org/10.3324/haematol.2021.278411

(C)2022 Ferrata Storti Foundation

Material published in Haematologica is covered by copyright. All rights are reserved to the Ferrata Storti Foundation. Use of published material is allowed under the following terms and conditions:

https://creativecommons.org/licenses/by-nc/4.0/legalcode. Copies of published material are allowed for personal or internal use. Sharing published material for non-commercial purposes is subject to the following conditions:

https://creativecommons.org/licenses/by-nc/4.0/legalcode, sect. 3. Reproducing and sharing published material for commercial purposes is not allowed without permission in writing from the publisher. 
existing ALL regimens, ${ }^{10}$ predicting the tolerability of new combination protocols has proven challenging without accurate information on how current conventional ALL therapy is actually administered.

Our aim was to tabulate the actual dosages and dose intensities of conventional chemotherapy in two consecutive multiagent front-line pediatric St. Jude ALL trials that used highly similar backbones but different formulations of asparaginase: E. coli L-asparaginase in Total 15 (T15) and pegaspargase in Total 16 (T16).

\section{Methods}

\section{Patients}

Patients were enrolled on St. Jude Children's Research Hospital protocols T15 (ClinicalTrials.gov ID: NCT00137111) ${ }^{11}$ and T16 (ClinicalTrials.gov ID: NCT00549848) ${ }^{12}$ for newly diagnosed ALL (Online Supplementary Figure S1). T15 and T16 therapy included remission induction therapy followed by consolidation therapy and 120 weeks of continuation therapy (146 weeks for boys on T15) which included two phases of reinduction (Online Supplementary Table S1). After remission induction, patients were classified for risk-adapted therapy as low-risk (LR, about $40 \%$ of patients), standard-risk (SR, about $50 \%$ of patients) or high-risk (HR, about $10 \%$ of patients). ${ }^{11,12}$ Patients with Down syndrome received altered methotrexate and leucovorin regimens, and HR patients received intensification phases, and thus both groups were excluded from this analysis. This report focuses on the $\sim 90 \%$ of patients who were treated on either the LR or SR arms. Ninety patients on T15 who received most of their therapy at a collaborating institution ${ }^{11}$ were also excluded, because their drug administration data were difficult to verify (Online Supplementary Figure S2). The studies were approved by the Institutional Review Board. Informed consent was obtained from either the parents or the patients, consistent with the Declaration of Helsinki.

\section{Treatment}

Drug administration data were recorded prospectively on protocol-specific forms, generally on a daily basis for induction and reinduction and on a weekly basis for other phases, by clinical staff and research nurses, and entered into centralized St. Jude databases by protocol-specific research data managers. Reasons for dose modifications were protocol-specified (Online Supplement).

Treatment regimens have been described previously ${ }^{1,112}$ and are summarized in Online Supplementary Figure S1 and Online Supplementary Table S1. The two protocols differed primarily by first-line asparaginase formulation, although there were a few other differences (Online Supplementary Figure S1, Online Supplementary Tables S1 and S2). ${ }^{11}$ Patients received E. coli asparaginase (Elspar) in T15, and pegaspargase (Oncaspar) in T16. In the case of allergic reaction to either $E$. coli asparaginase or pegaspargase, an asparaginase formulation change was permitted by the protocol (Online Supplement). To compare planned dosages roughly between protocols, Elspar doses were converted to comparable doses of pegaspargase based on protocol-spec-

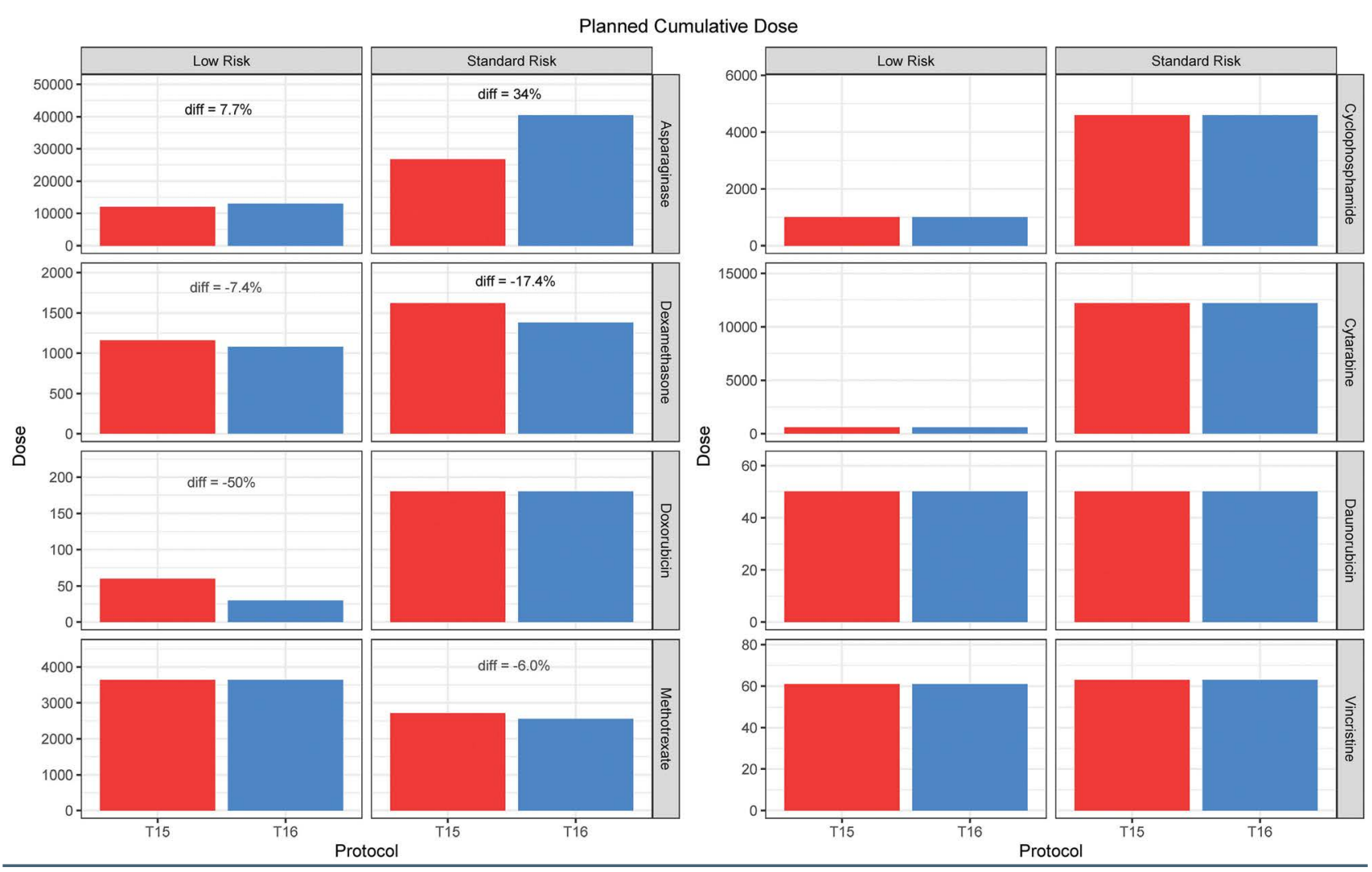

Figure 1. Planned cumulative doses of drugs used in the low- and standard-risk arms of T15 and T16. The only drugs with at least a $10 \%$ difference in planned cumulative dosages between T16 and T15 (within risk arms) were asparaginase (34\% higher in the standard risk arm), dexamethasone (17.4\% lower in the standard-risk arm), and doxorubicin (50\% less in the low-risk arm). Thus, only asparaginase was planned for higher dosages on T16 than on T15. There were no differences between T15 and T16 in planned cumulative dosages of prednisone, vincristine, daunorubicin, cytarabine, cyclophosphamide, mercaptopurine; there was a planned decrease of only $6.0 \%$ in low-dose methotrexate dosages in the standard-risk arm on T16 versus T15. See Online Supplementary Table S2 for details on planned dosages. 
ified conversions (i.e., pegaspargase 2,500 units $/ \mathrm{m}^{2}$ were considered comparable to 50,000 units $/ \mathrm{m}^{2}$ of Elspar and 100,000 units $/ \mathrm{m}^{2}$ of Erwinia asparaginase). Total planned dosages were identical for T15 LR and T16 LR as well as for T15 SR and T16 SR for consolidation high-dose methotrexate, cyclophosphamide, cytarabine, daunorubicin, continuation mercaptopurine, and vincristine; they were higher on T16 than on T15 for asparaginase, and were lower on T16 than on T15 for dexamethasone (both LR and SR arms) and for doxorubicin (LR arms; Figure 1, Online Supplementary Table S2). In Total therapy protocols, particularly for the 120 weeks of continuation, dosages of therapy were adjusted or omitted if they caused toxicity (particularly myelosuppression), as detailed (Online Supplementary Table S2), but therapy was not typically delayed.

Dose intensities were calculated as the delivered dosage divided by the protocol-specified dosage for each risk group and protocol. Dose intensity was tabulated per phase, and cumulative dose intensity was estimated as the total prescribed dosage per patient divided by the total cumulative protocol-specified dosage (Online Supplement). Absolute neutrophil count (ANC) was generally checked at least weekly throughout treatment (Online Supplement). Time to complete therapy was the time from start of first chemotherapy in induction to last dose of chemotherapy at 120 weeks.

\section{Additional methods}

Details of genetic ancestry assessment, TPMT phenotype assignment, and statistical methods are available in the Online Supplement.

\section{Results}

Differences in dosage intensity and delivered dosages between the Total 15 and Total 16 trials

The median cumulative dose intensity for each drug ranged from $61 \%$ to $100 \%$, with the largest interpatient variability observed for mercaptopurine and methotrexate. Post-induction, dose intensity median absolute deviations ranged from $16 \%$ to $23 \%$ for mercaptopurine (Online Supplementary Table S3). The cumulative dose intensities for several drugs were higher on T15 than on T16 (Figure 2, Online Supplementary Table S3), and median cumulative dose intensities were over 10\% higher on T15 than on T16 for cyclophosphamide $(P<0.0001$ for SR patients), cytarabine $(P<0.0001$ for SR patients), mercaptopurine $(P<0.0001$ for LR and SR patients), and consolidation methotrexate $(P<0.0001$ for LR patients). Examining phases instead of cumulative dose intensity, mercaptopurine exhibited the lowest dose intensities of any drug in all protocols and arms, and its dose intensity was higher on T15 than on T16 for all phases $(P<0.0001$ for all) except induction (Online Supplementary Table S4). The dose intensity of cyclophosphamide was higher on T15 than on T16 during the continuation phase $(P<0.0001)$, while that of cytarabine was higher at reinduction II and during continuation $(P<0.0001$ for both phases; Online Supplementary Table S5 and S6).

Tolerated dosages of mercaptopurine were lower on T16 than they had been on T15, with as much as $27 \%$ higher dose intensity on T15 than on T16 (Figure 3, Online

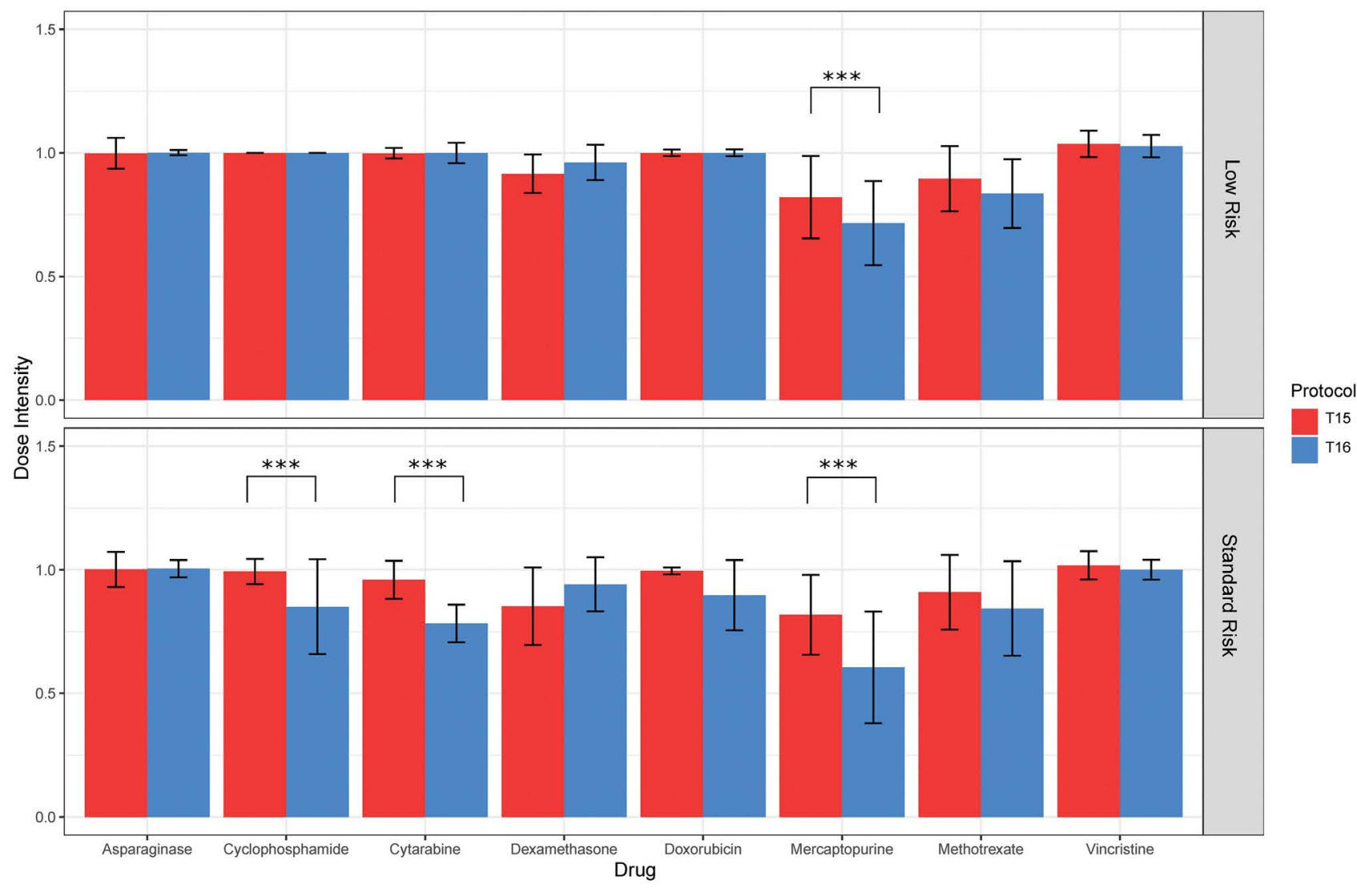

Figure 2. Administered cumulative dose intensities for drugs on T15 versus T16, by risk arm. The only significant differences ( $* * * P<0.0001$ ) in dose intensities with more than a $10 \%$ difference between protocols were for cyclophosphamide and cytarabine (standard-risk arms) and for mercaptopurine (both risk arms), all of which were higher on T15 than on T16 (see Online Supplementary Table S3 for details on all drugs). Bars and whiskers indicate medians and median absolute deviations among each patient population. There was a total of 16 statistical comparisons, thus the Bonferroni significance threshold=0.003. 

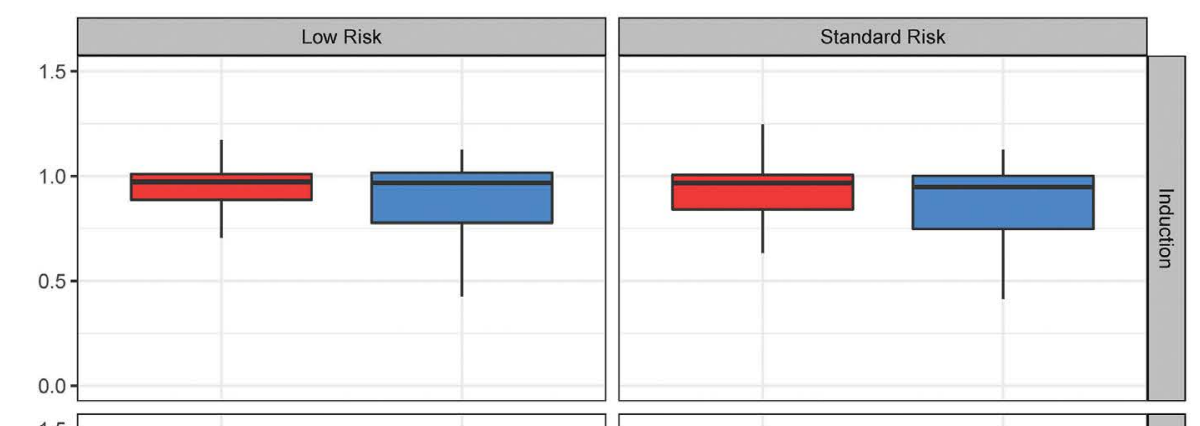

Figure 3. Thiopurine dose intensity by phase in T15 versus T16. The box and whisker plots show the quartiles and nonoutlier ranges. Dose intensity was significantly lower on T16 than on T15 $(P<0.0001)$ for all phases except induction (see Online Supplementary Table S4 for exact $P$ values). The largest difference was $27 \%$ during continuation weeks 10 16 in standard-risk patients. There was a total of 10 statistical comparisons, thus the Bonferroni signifi-
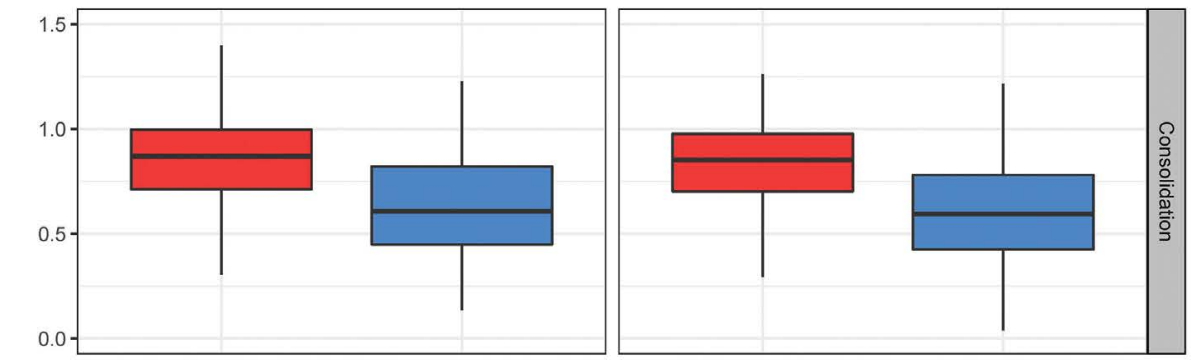
cance threshold $=0.005$.
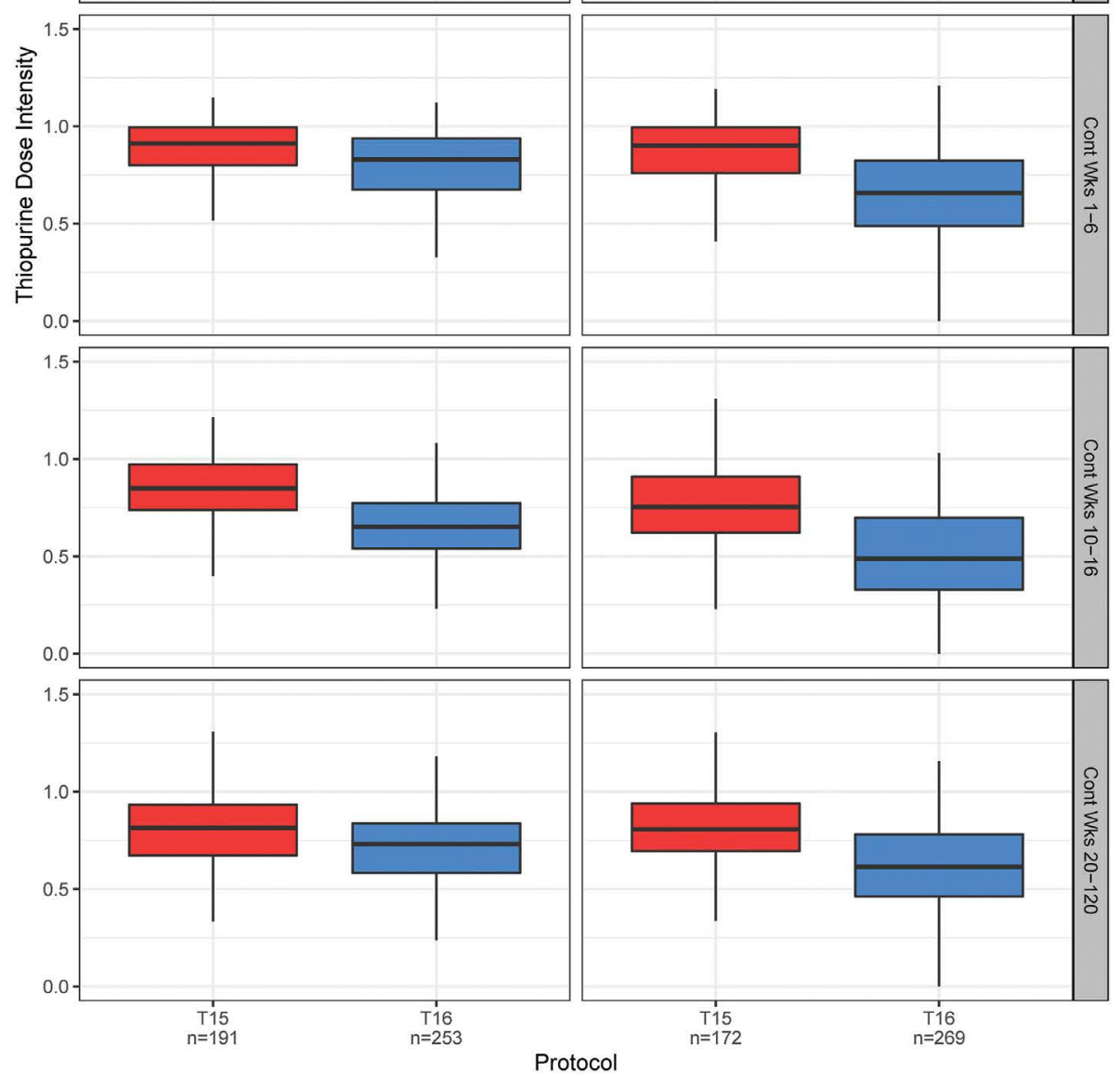

Supplementary Table S4). The median cumulative dosage of mercaptopurine delivered was $7,420 \mathrm{mg} / \mathrm{m}^{2}$ higher on the LR arm and $9,130 \mathrm{mg} / \mathrm{m}^{2}$ higher on the SR arm of T15 compared to T16 (Table 1). Despite these markedly higher dosages on T15 (Table 1), ANC did not differ between the protocols for the LR arm $(P=0.18)$ (Figure 4), and were actually lower on T16 than on T15 for the SR arm $(P<0.0001)$, indicating that our clinicians were not titrating to a higher ANC on T16 than they had on T15. Interestingly, the only drug for which dose intensity was significantly higher on T16 than on T15 was dexamethasone $(P<0.0001)$ (Online Supplementary Table S3), although the administered cumula-

tive dosage was higher on T15 than on T16, reflecting the fact that the planned dosage (i.e., the denominator) was lower on T16 than on T15.

For most drugs on each of the LR and SR arms, the planned dosages were identical on T15 and T16 (Figure 1, Online Supplementary Table S2); the drug with the largest planned differences in dosages between the two protocols was asparaginase, with an 8\% planned increase in the LR arms and a 34\% increase in the SR arms on T16 compared to T15. In actuality, asparaginase was the only drug for which the planned or administered dosages were higher on T16 than on T15, with higher cumulative dosages given on 
Table 1. Actual cumulative dosages administered for drugs on T15 and T16 by risk group.

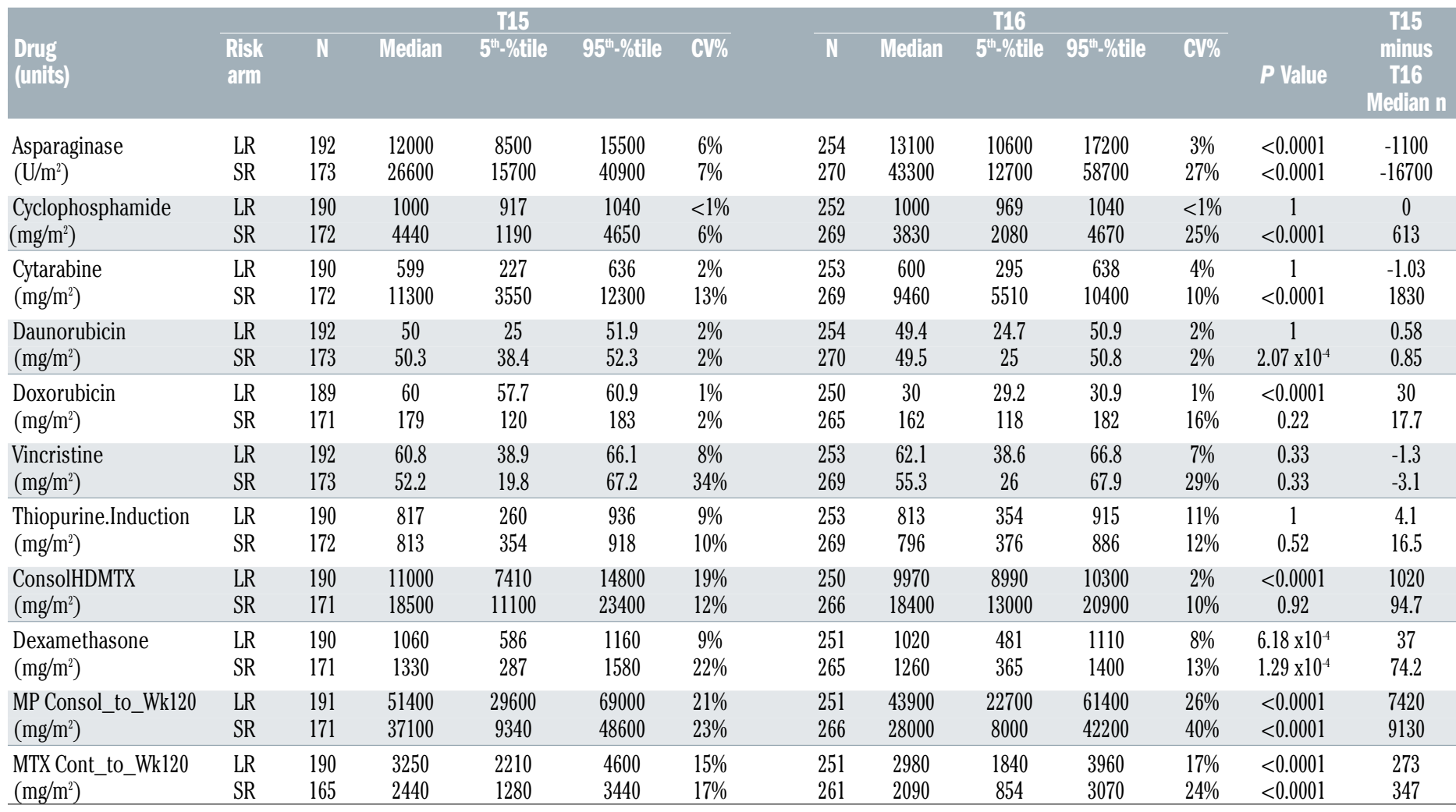

Drugs excluded were prednisone from the induction phase (because of variability in formulations and documentation) and mercaptopurine and methotrexate for boys in T15 from week 120 146 (which were not present in T16). The CV\% is calculated as median absolute deviation divided by median dosage. Total of 11 (drugs)x2(risk arms)=22 comparisons. Bonferroni significance

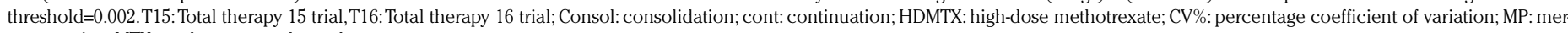
captopurine; MTX: methotrexate; wk: week.

T16 than on T15 for both the LR and SR arms $(P<0.0001$ for both) (Table 1). The higher exposure was due to the planned higher dosages on T16 than on T15, rather than to a change in prescribing practices, in that the dose intensities for asparaginase did not differ on T16 versus T15 for either the LR $(P=1)$ or the SR $(P=0.77)$ arms (Online Supplementary Table S3).

The dose intensity for mercaptopurine was higher in those who had antibodies against asparaginase (against Elspar for T15 and against pegaspargase for T16) than in those who did not $(P=0.12$ for T15 LR, $P=0.0056$ for T15 SR, $P=0.00027$ for T16 LR, and $P=0.00014$ for T16 SR) (Figure 5). The higher dose intensity was likely in response to a higher ANC in those with antibodies compared to those without antibodies, with the difference only reaching nominal statistical significance for T15 SR $(P=0.032)$ (Figure $5)$.

\section{Differences in dose intensity by inherited genetics and other characteristics of the patients (age, sex, race)}

The only dosage modification based on a pharmacogenetic characteristic for both protocols was that for thiopurines based on TPMT status (Online Supplementary Table S2), and this modification was made prospectively. Thus, as expected, the median dose intensity was lower for those with a TPMT abnormality (poor or intermediate metabolizers) than for those without a defective TPMT allele (normal metabolizers): 0.73 versus 0.83 for T15 SR, 0.68 versus 0.84 for T15 LR, 0.48 versus 0.63 for T16 SR, and 0.60 versus 0.75 for T16 LR $\left(P=0.001, P=4.3 \times 10^{-4}, P<0.0001\right.$, and $P=1.92 \times 10$ ${ }^{4}$, respectively) (Online Supplementary Figure S3, Online
Supplementary Table S7). Importantly, using this prospective precision medicine approach of genetically-driven prescribing prevented excessive thiopurine-induced cytopenias. As a result, neither the dose intensities of other drugs (anthracyclines, asparaginase, methotrexate, cyclophosphamide, cytarabine, and dexamethasone) (Online Supplementary Table S7) nor the ANC (Figure 6) differed (or differed only marginally) by TPMT status, demonstrating that pinpointing the correct drug for dosage adjustments prevented compromising the dosages of other chemotherapeutic agents.

When mercaptopurine dose intensity was re-estimated using a TPMT-specific denominator for expected mercaptopurine dosages (see the Online Supplement for details), the dose intensity for mercaptopurine showed a much smaller difference between those with and those without a defect in TPMT (Online Supplementary Figure S4) than when the denominator was not adjusted downward for the expected decrease in dosage due to TPMT status (Online Supplementary Figure S5).

We compared dose intensity for all drugs by sex, ancestral group (white, Black, Hispanic, and other), and age, adjusting for protocol and risk arm. Differences in dose intensity by race and by sex were relatively modest (data not shown), particularly compared to the differences by protocol or risk group. There were a few agents for which dose intensity differed by age (Online Supplementary Figure S6). For T15 SR, T16 SR, and T16 LR, the dose intensities for dexamethasone and vincristine were significantly inversely correlated to age and the dose intensity for methotrexate was positively correlated to age; for T15 LR, only the dose intensity of vincristine was inversely correlated with age, 


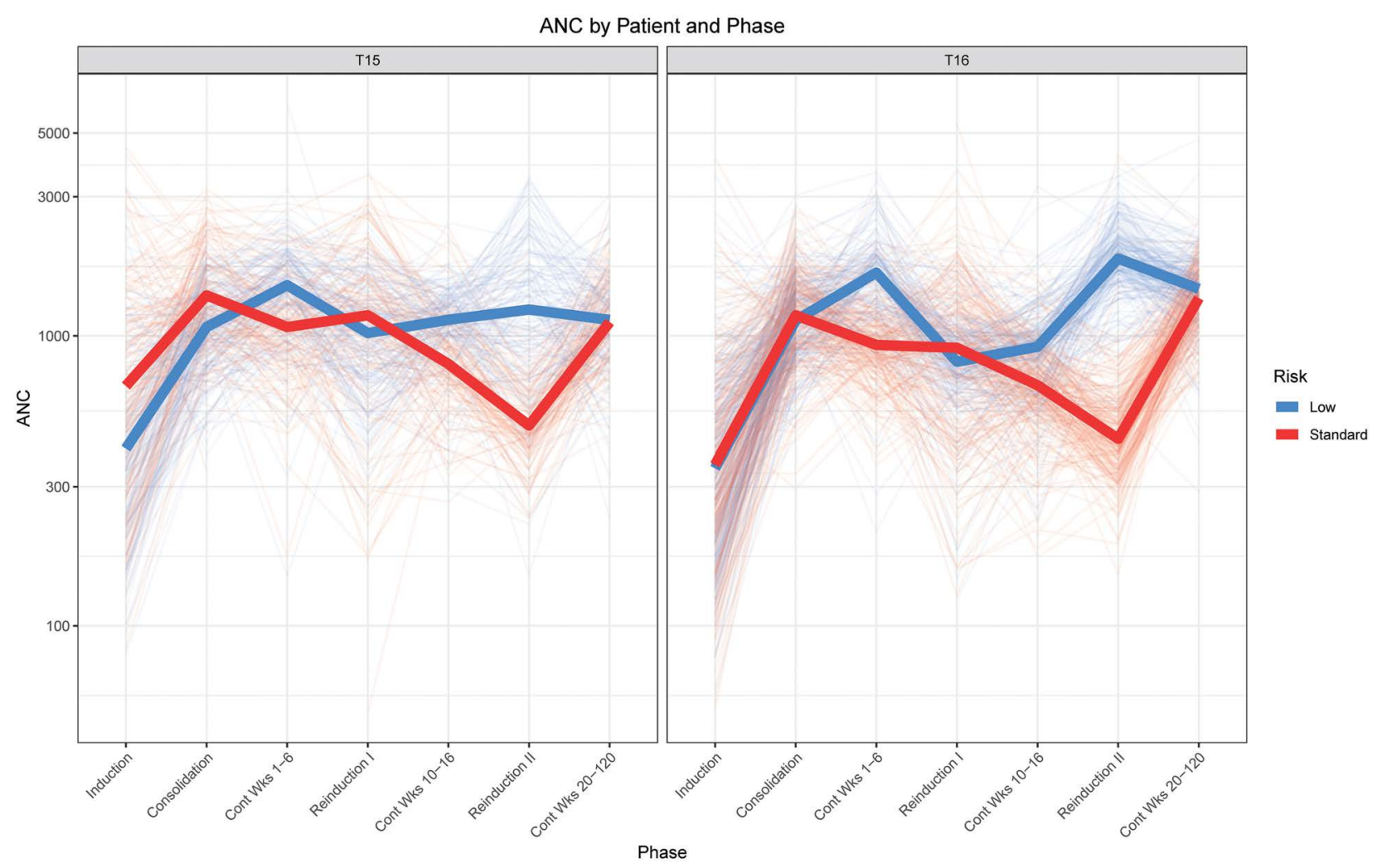

Figure 4. Absolute neutrophil count by risk group on T15 versus T16. The graphs show the average of fitted absolute neutrophil count (ANC; cells/mm ${ }^{3}$ ) data per phase per patient with thick solid lines representing the median per risk group. Based on 46,310 and 64,549 ANC records for T15 and T16, respectively.

but the effect did not reach statistical significance after correction for multiple testing.

\section{Differences in dose intensity between risk arms}

The most striking difference in dose intensity between risk arms was for mercaptopurine (Figure 2). The median dose intensity was similar between risk groups for most phases on T15 (Online Supplementary Table S8) including the phase immediately after Elspar asparaginase ended (weeks $20-47, P=0.1$ ). In contrast, on T16, in the weeks immediately following reinduction and the completion of pegaspargase (which ended week 29), the median dose intensity was $41 \%$ versus $69 \%$ for the SR versus the LR arms $(P<0.0001)$ (Online Supplementary Table S9). The cumulative dose intensities for all the drugs by protocol and risk arm are shown in Online Supplementary Tables S10 and S11.

\section{Relapse}

We explored relationships between dose intensities for all drugs during all phases and cumulatively, and ANC for all phases and cumulatively, and treatment outcomes. No significant associations between dose intensity or ANC and outcomes were observed after adjusting for multiple testing. Only one dose intensity or ANC variable was nominally associated with outcome in the same direction for both protocols in both continuous and tertile analyses: for those who finished 120 weeks of therapy: a higher dose intensity for mercaptopurine was nominally associated with a higher risk of any relapse (unadjusted $P=0.03$ for T15, $P=0.07$ for T16, tertile analysis) (Online Supplementary Table S12, Online Supplementary Figure S7). For T15, this tendency for an association between higher mercaptopurine dose intensity and worse outcomes was also true when including patients who did not complete 120 weeks of therapy (Online Supplementary Table S12). Associations between ANC and outcomes were not reproducible across phases or protocols. Notably, there was no association between dose intensities of any other medications, including asparaginase (Online Supplementary Figure S8) and outcomes; however, it should be noted that there was also little interpatient variability in the dose intensity for asparaginase (median absolute deviations, 1\% to 7\%; Online Supplementary Table S3).

\section{Absolute neutrophil count versus dose intensity}

For each drug, protocol, and risk arm, we analyzed whether dose intensity was related to ANC for each phase. The strongest associations were for mercaptopurine; in all instances with nominal associations, ANC and dose intensity were positively correlated (Online Supplementary Table S13). This reflects that clinicians followed protocol recommendations (Online Supplementary Table S1) to increase the mercaptopurine dosage in those with high ANC, and to decrease the dosage for those with low ANC. For all associations between dose intensity of other drugs and ANC, the correlations were also positive, with a few exceptions; dexamethasone dose intensity was inversely correlated with ANC in some phases (although not statistically significantly after corrections for multiple testing; data not shown).

\section{Dose intensity versus time on therapy}

Although the practice was to avoid delays in therapy, there was observed variability in the time required to complete all therapy up to week 120 of continuation (median time to complete therapy including induction, consolida- 
tion, and continuation was 135.5 weeks for both T15 and T16; interquartile range, 134.4-137.3 weeks). To assess the possible impact of delivered dose intensity on time to complete therapy, we analyzed the association between the dose intensity of asparaginase and mercaptopurine and time to complete therapy in patients completing treatment. We found an inverse association between dose intensity and time to complete therapy $(P<0.0001$ for both drugs), however, the correlation was relatively weak $\left(r^{2}=0.03\right.$ for both asparaginase and mercaptopurine). More importantly, there was no association between time to complete therapy and relapse risk $(P=0.7$ on T15, $P=0.4$ on T16). Of the 889 patients included on the study, $17(1.9 \%)$ discontinued treatment early due to toxicity. With a median follow-up of 7.5 years from diagnosis for these patients, only one of 17 patients $(5.9 \%$ ) experienced a relapse (at 3.9 years after diagnosis).

\section{Discussion}

This study is the first to comprehensively evaluate dosages of all conventional drugs constituting modern ALL therapy. Our most striking observation was that mercaptopurine dose intensity was lower on T16 than on T15, despite no planned changes to mercaptopurine dosages on T16 versus T15. The most likely explanation for this decreased ability to administer full dosages of mercaptopurine is higher planned and administered doses of asparaginase on T16 than on T15. We suggest that asparaginase interfered with mercaptopurine delivery based on several findings: an inverse association between delivered asparaginase and mercaptopurine dosages between protocols (Figure 2, Table 1, Online Supplementary Table S4), a higher delivered mercaptopurine dosage in those with antibodies to asparaginase than in those without antibodies (Figure 5), and a temporal decrease in dose intensity of mercaptopurine that corresponds with the timing of asparaginase use (Online Supplementary Tables S8 and S9, Online Supplementary Figures S4 and S5). Moreover, we and others have shown that asparaginase can decrease the clearance of other drugs, such as dexamethasone, putatively through its hypoproteinemic effects on hepatic drug metabolizing enzymes and transporters. We found that this interaction is associated with an increased risk of at least one adverse effect of dexamethasone (osteonecrosis) and higher drug exposure both in the clinic ${ }^{13}$ and in preclinical models. ${ }^{14}$ Others have also hypothesized that asparaginase can influence the dose intensity or toxicity of thiopurines and/or methotrexate..$^{15,16}$

Asparaginase is an important part of ALL therapy, which is the reason that we chose to increase exposure to asparaginase on T16 compared to that on T15. Early discontinuation of asparaginase was associated with lower event-free survival in the Dana-Farber 91-01 study ${ }^{1}$ and in ETV6/RUNX1 ALL, ${ }^{17}$ and patients treated with E. coli asparaginase had fewer relapses than those treated with Erwinase..$^{18}$ Patients with allergy to pegaspargase treated on recent Children's Oncology Group protocols who did not receive all asparaginase therapy had a lower disease-free survival, and drug shortages of Erwinase contributed to not being able to compensate fully for missed pegaspargase doses. ${ }^{19}$ However, prolonged asparaginase did not improve outcomes in two trials,, 20 and higher doses of pegaspargase did not improve outcome relative to standard doses..$^{12}$ In the
T15

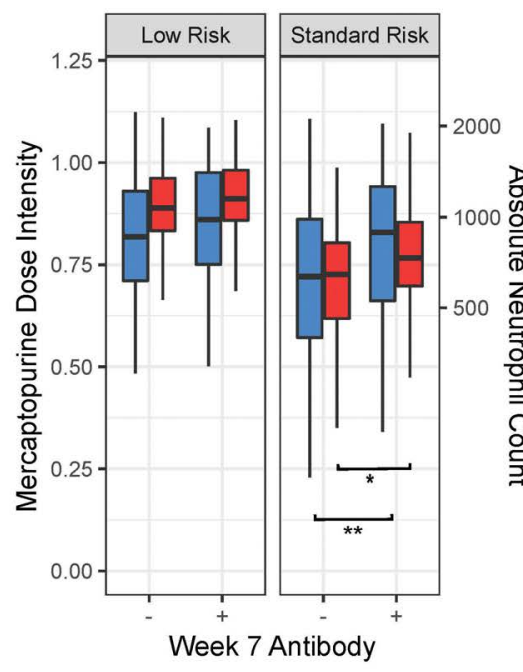

T16

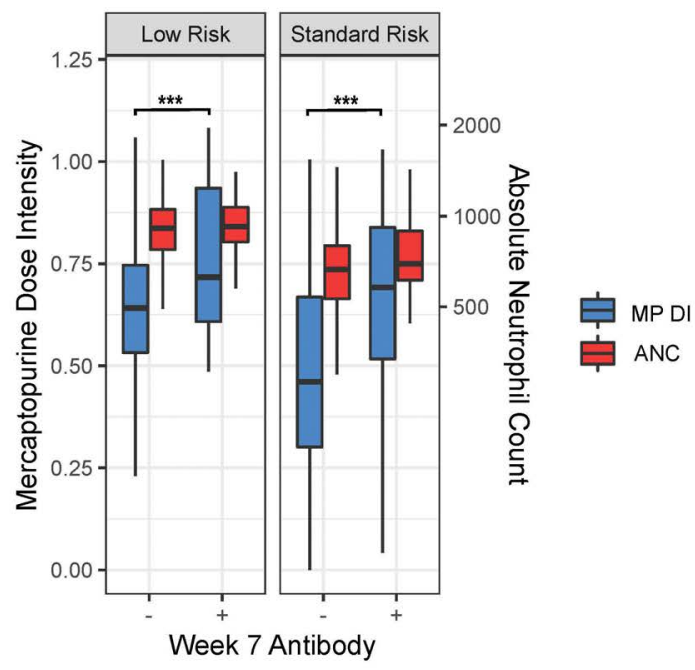

Figure 5. Mercaptopurine cumulative dose intensity and absolute neutrophil count in patients negative or positive for anti-asparaginase antibodies. Mercaptopurine cumulative dose intensity (DI; left y axes) and absolute neutrophil count (ANC) in cells $/ \mathrm{mm}^{3}$ (right y axes) for continuation weeks 10-16 in patients who were negative or positive for anti-asparaginase antibodies against Elspar (T15) or Oncaspar (T16) measured at continuation week 7. Boxes and whiskers represent quartiles and non-outlier ranges. Nominal $P$ values were $\star P<0.05 ; * * P<0.01, * * * P<0.001$. There was a total of four comparisons, thus the Bonferroni significance threshold $=0.01$

current analysis, we did not find that asparaginase dose intensity was related to disease free-survival in either T15 (using primarily native E. coli asparaginase) or T16 (using primarily pegaspargase) (Online Supplementary Table S12, Online Supplementary Figure S8). There are several possible explanations for this finding. First, we used more asparaginase on our studies than others did, thus perhaps exceeding some threshold value for exposure, consistent with the lack of influence of pegaspargase dosage on relapse in T16. ${ }^{12}$ Second, in those with allergy to their front-line asparaginase preparation, substitution with another formulation was aggressive, such that asparaginase dose intensity was not lower for those with or without allergy on T15 or on T16 (Online Supplementary Figure S9) and interpatient variability in dose intensity for asparaginase was low, i.e., $<7 \%$ (Online Supplementary Table S3). Another possibility is that even in those patients with relatively low asparaginase dose 

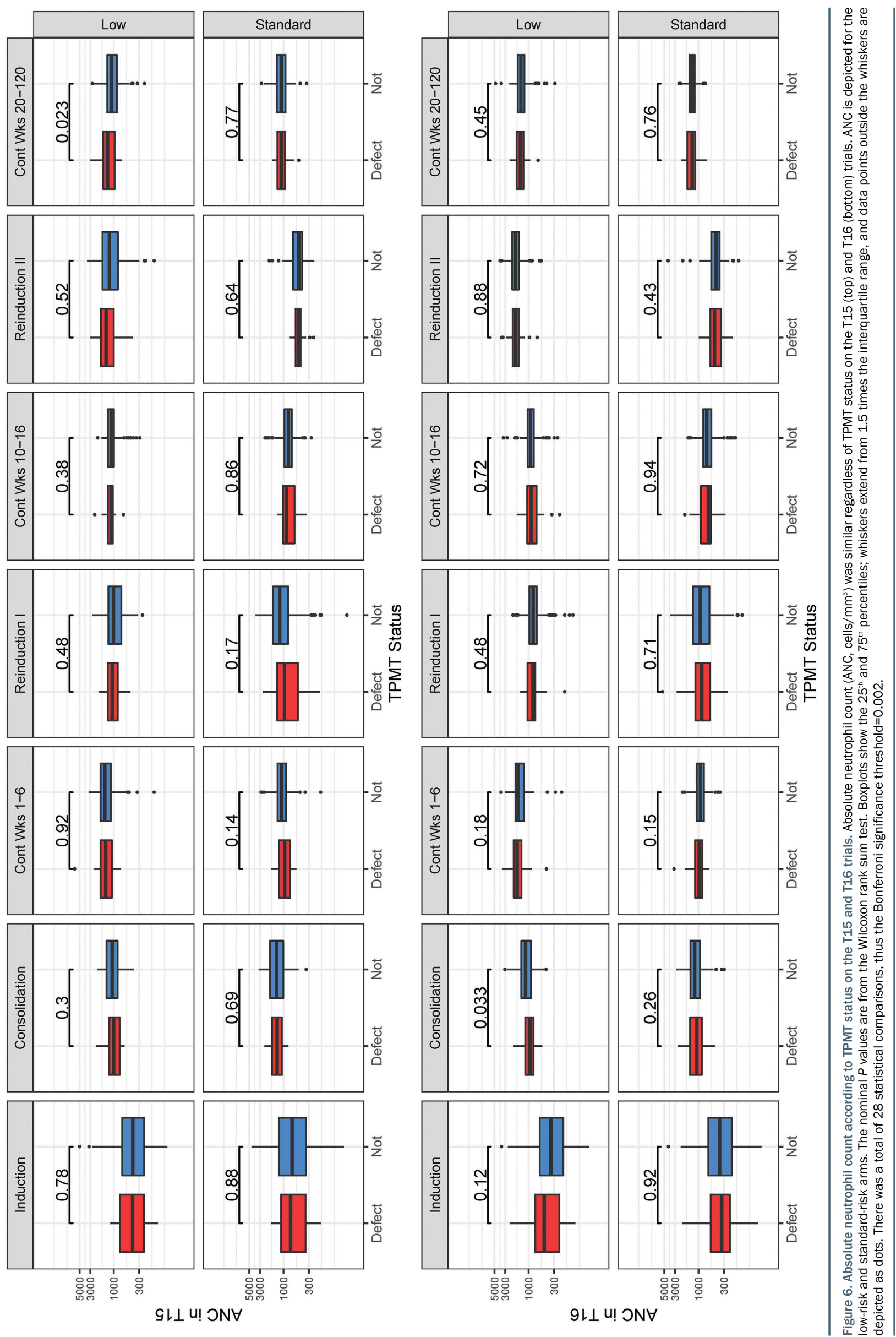
intensity, the dosages of other medications were increased, and this could have compensated for the lower exposure to asparaginase. In fact, we found that patients with low asparaginase dose intensity had significantly higher dose intensity for methotrexate on T16 (Online Supplementary Figure S10), consistent with our protocol recommendations for substituting methotrexate in those who could not receive asparaginase due to allergy or pancreatitis. These findings represent what is unique about the current study, because we have data to indicate how dosages of each medication may have influenced each other. This is the situation clinicians face: when pressing ahead with one medication is thwarted for some reason (drug shortages, adverse effects), other medications are often substituted. In these trials, it appears that the substitutions made were effective in maintaining efficacy, in that there were no associations between low exposure to any one drug and outcome.

In our studies, as in other ALL studies, the primary drugs for which dosage adjustments are routinely made are mercaptopurine and methotrexate, which are titrated to a desired ANC, and indeed the greatest variability in dosages was observed for these two drugs (Online Supplementary Table S3). There is controversy as to whether increasing the dose intensity of mercaptopurine and/or methotrexate increases, ${ }^{21}$ decreases ${ }^{22}$ or has no effect ${ }^{16}$ on the risk of relapse. Complicating the interpretation of the data is that most studies do not systematically assess adherence, and most protocols use both drugs orally; thus, patients with the highest prescribed dose intensity may be the patients who are actually taking the lowest percentage of their therapy, thereby complicating any interpretation of how dose intensity affects relapse. For example, those with lower measured mercaptopurine adherence had higher relapse risk, but there was no association with mercaptopurine dose intensity ${ }^{23}$ It is possible that low adherence to mercaptopurine also translates into lower adherence with other drugs not measured (e.g., glucocorticoids, methotrexate, supportive care drugs), thus compounding the risk of relapse due to poor adherence. In the current analysis, dosages of all antileukemic drugs were captured, and the only drug whose prescribed dose intensity was associated with relapse in unadjusted analyses on both T15 and T16 was mercaptopurine (Online Supplementary Table S12, Online Supplementary Figure S7), albeit not in the same phases of therapy for both studies. It should be noted that the direction of association was that higher dose intensity was associated with higher relapse, indicating that it is likely that high dose intensity identified patients who were either noncompliant (and thus clinicians needed to push the dosage to achieve desired neutropenia) or had very fast drug clearance. However, it should be noted than no metrics of dose intensity or of ANC were significantly associated with relapse or outcome using $P$ value thresholds adjusted for the large number of exploratory comparisons. Although our data comprehensively capture prescribed mercaptopurine dose, one limitation of these data is that we did not assess adherence to administration of prescribed drug in patients who were not under direct medical supervision, which applied to most of the oral doses of glucocorticoids and mercaptopurine. Any association between dose intensity and outcomes is likely to be affected by the extent to which adherence is emphasized, the extent of thiopurine monitoring, and the rigor with which ANC targets are pursued. It should be noted that on T15, there was a modest association between higher ANC and increased relapse, but this association was not statistically significant after correction for multiple testing, and was not reproducible, as no such association was observed on T16, and in subanalyses for different therapy phases, higher ANC tended to associate with both increased and decreased relapse.

In both our studies, thiopurine starting dose was adjusted based on TPMT status, ${ }^{24}$ which appropriately resulted in lower dosages of mercaptopurine in those with a genetic defect in TPMT; herein, we show for the first time that this allowed for uncompromised dosing of the other chemotherapeutic agents (Online Supplementary Table S7) and no difference in ANC based on TPMT status (Figure 6).

It is also worth noting that the dose intensity for mercaptopurine, especially in the first 6 months of therapy, was lower than reported by others; ${ }^{23}$ after reinduction, the median dose intensity was as low as $49 \%$ for one phase on T16, suggesting that our planned dosages may have been too high (Online Supplementary Table S4). Interestingly, the median dose intensity for the comparable time period on T15, when native asparaginase rather than pegaspargase was used, was higher at $76 \%$. Had we realized a priori the impact of asparaginase on patients' tolerance of thiopurine therapy, we could have designed a more realistic dosage regimen, and this finding has implications for future protocol design.

There are conflicting data on the importance of "intensive" non-antimetabolite therapy in ALL., ${ }^{3,-7,25-29}$ Although the lack of association of outcomes with dose intensity for most drugs in our study is fairly consistent with studies touting deintensification strategies, St. Jude differs from many other centers in that patients receive all weekly methotrexate parenterally (and thus return to the clinic every week, in contrast to many centers that see patients only every 4-6 weeks during continuation), LR and SR patients received vincristine/dexamethasone pulses throughout continuation, and a high percentage of patients $(\sim 50 \%)$ received therapy on the SR and HR arms, which include more asparaginase and other non-antimetabolite agents (cytarabine, cyclophosphamide) than many other treatment protocols. Thus, the lack of association between dose intensity and relapse we observed may not be extensible to centers with less intensive monitoring and/or less chemotherapy-dense and diverse regimens. It also suggests that, for patients intolerant to specific components of therapy (e.g., asparaginase due to pancreatic or hepatic toxicity), substitution with alternative chemotherapy may mitigate the adverse prognosis associated with early discontinuation of the offending agent. Given that therapy-limiting toxicities may preclude delivery of prescribed chemotherapy in $12-25 \%$ of patients receiving treatment on modern trials, ${ }^{19}$ prospective evaluation of chemotherapy substitution to address therapy-limiting toxicity should be considered.

We conclude that intentional changes to the dose intensity of one agent, e.g., asparaginase, can have dramatic consequences on the ability to administer other conventional agents. Comprehensive data on chemotherapy actually delivered in cancer clinical trials are needed to fully interpret results and further optimize therapy.

\section{Disclosures}

MVR and HI and St. Jude Children's Research Hospital receive investigator-initiated research funding from Servier Pharmaceutical. 


\section{Contributions}

SEK, WEE, CHP, SJ and MVR conceived and designed the study; KRC, ARM, CC, DP, SEK, HI, JER, MLM, RCR, TAG, JJY, WEE, SJ, CHP, and MVR provided study material or patients and collected and/or assembled data; NMK, YL, $J C P, E R F, C A S, W Y, C C, Y L$ and DP, analyzed and interpreted data; MVR wrote the first draft of the manuscript; all authors contributed to the revision of the manuscript; and all authors approved the final submitted version of the manuscript.

\section{Acknowledgments}

The authors thank the patients and parents who participated in the clinical protocols included in this study, and the participating clinicians and research staff.

\section{Funding}

This work was supported by National Institutes of Health GM115279, CA35401, CA142665, CA21765, and K08CA250418, and the American Lebanese Syrian Associated Charities. The content is solely the responsibility of the authors and does not necessarily represent the official views of the National Institutes of Health.

\section{Data-sharing statement}

De-identified data used in the preparation of this manuscript are available upon request.

\section{References}

1. Silverman LB, Gelber RD, Dalton VK, et al. Improved outcome for children with acute lymphoblastic leukemia: results of DanaFarber Consortium Protocol 91-01. Blood. 2001;97(5):1211-1218.

2. Albertsen BK, Grell K, Abrahamsson J, et al. Intermittent versus continuous PEGasparaginase to reduce asparaginase-associated toxicities: a NOPHO ALL2008 randomized study. J Clin Oncol. 2019;37(19): 1638-1646

3. Moricke A, Zimmermann M, Reiter A, et al. Long-term results of five consecutive trials in childhood acute lymphoblastic leukemia performed by the ALL-BFM study group from 1981 to 2000 . Leukemia. 2010:24(2):265-284

4. Smid EJ, Driessen AJ, Konings WN. Mechanism and energetics of dipeptide transport in membrane vesicles of Lactococcus lactis. J Bacteriol. 1989;171(1): 292-298.

5. Veerman AJ, Kamps WA, van den Berg H, et al. Dexamethasone-based therapy for childhood acute lymphoblastic leukaemia: results of the prospective Dutch Childhood Oncology Group (DCOG) protocol ALL-9 (1997-2004). Lancet Oncol. 2009;10(10): 957-966.

6. Matloub Y, Bostrom BC, Hunger SP, et al. Escalating intravenous methotrexate improves event-free survival in children with standard-risk acute lymphoblastic leukemia: a report from the Children's Oncology Group. Blood. 2011;118(2):243-251.

7. Seibel NL, Steinherz PG, Sather HN, et al. Early postinduction intensification therapy improves survival for children and adolescents with high-risk acute lymphoblastic leukemia: a report from the Children's Oncology Group. Blood. 2008;111(5):25482555.

8. Boissel N, Baruchel A. Acute lymphoblastic leukemia in adolescent and young adults: treat as adults or as children? Blood. 2018;132(4):351-361

9. Gaynon PS, Steinherz PG, Bleyer WA, et al. Association of delivered drug dose and outcome for children with acute lymphoblastic leukemia and unfavorable presenting features. Med Pediatr Oncol. 1991;19(4):221227.

10. Kantariian H, Ravandi F, Short NJ, et al. Inotuzumab ozogamicin in combination with low-intensity chemotherapy for older patients with Philadelphia chromosomenegative acute lymphoblastic leukaemia: a single-arm, phase 2 study. Lancet Oncol. 2018:19(2):240-248.

11. Pui $\mathrm{CH}$, Campana $\mathrm{D}$, Pei $\mathrm{D}$, et al. Treating childhood acute lymphoblastic leukemia without cranial irradiation. N Engl J Med. 2009;360(26):2730-2741.

12. Jeha S, Pei D, Choi J, et al. Improved CNS control of childhood acute lymphoblastic leukemia without cranial irradiation: St Jude Total Therapy Study 16. J Clin Oncol. 2019:37(35):3377-3391

13. Kawedia JD, Liu C, Pei D, et al. Dexamethasone exposure and asparaginase antibodies affect relapse risk in acute lymphoblastic leukemia. Blood. 2012;119(7): 1658-1664.

14. Liu C, Janke LJ, Kawedia JD, et al. Asparaginase potentiates glucocorticoidinduced osteonecrosis in a mouse model. PLoS One. 2016;11(3):e0151433.

15. Merryman R, Stevenson KE, Gostic WJ 2nd, et al. Asparaginase-associated myelosuppression and effects on dosing of other chemotherapeutic agents in childhood acute lymphoblastic leukemia. Pediatr Blood Cancer. 2012;59(5):925-927.

16. Chessells JM, Harrison G, Lilleyman JS, Bailey CC, Richards SM. Continuing (maintenance) therapy in lymphoblastic leukaemia: lessons from MRC UKALL X. Medical Research Council Working Party in Childhood Leukaemia. Br J Haematol. 1997;98(4):945-951.

17. Usami I, Imamura T, Takahashi Y, et al. Discontinuation of L-asparaginase and poor response to prednisolone are associated with poor outcome of ETV6-RUNX1positive pediatric B-cell precursor acute lymphoblastic leukemia. Int J Hematol. 2019;109(4):477-482

18. Duval M, Suciu S, Ferster A, et al. Comparison of Escherichia coli-asparaginase with Erwinia-asparaginase in the treatment of childhood lymphoid malignancies: results of a randomized European Organisation for Research and Treatment of Cancer-Children's Leukemia Group phase 3 trial. Blood. 2002;99(8):2734-2739.

19. Gupta S, Wang C, Raetz EA, et al. Impact of asparaginase discontinuation on outcome in childhood acute lymphoblastic leukemia: a report from the Children's Oncology Group. J Clin Oncol. 2020;38(17):1897-1905

20. Mondelaers V, Suciu S, De Moerloose B, et al. Prolonged versus standard native E. coli asparaginase therapy in childhood acute lymphoblastic leukemia and non-Hodgkin lymphoma: final results of the EORTCCLG randomized phase III trial 58951.
Haematologica. 2017;102(10):1727-1738.

21. Schmiegelow K, Nielsen SN, Frandsen TL, Nersting J. Mercaptopurine/methotrexate maintenance therapy of childhood acute lymphoblastic leukemia: clinical facts and fiction. J Pediatr Hematol Oncol. 2014;36 (7):503-517.

22. Relling MV, Hancock ML, Boyett JM, Pui $\mathrm{CH}$, Evans WE. Prognostic importance of 6 mercaptopurine dose intensity in acute lymphoblastic leukemia. Blood. 1999;93(9): 2817-2823

23. Bhatia S, Landier W, Hageman L, et al Systemic exposure to thiopurines and risk of relapse in children with acute lymphoblastic leukemia: a Children's Oncology Group study. JAMA Oncol. 2015;1(3):287-295

24. Relling MV, Schwab M, Whirl-Carrillo M, et al. Clinical Pharmacogenetics Implementation Consortium guideline for thiopurine dosing based on TPMT and NUDT15 genotypes: 2018 update. Clin Pharmacol Ther. 2019;105(5):1095-1105.

25. Vora A, Goulden N, Mitchell C, et al Augmented post-remission therapy for a minimal residual disease-defined high-risk subgroup of children and young people with clinical standard-risk and intermediate-risk acute lymphoblastic leukaemia (UKALL 2003): a randomised controlled trial. Lancet Oncol. 2014;15(8):809-818.

26. Lange BJ, Bostrom BC, Cherlow JM, et al Double-delayed intensification improves event-free survival for children with intermediate-risk acute lymphoblastic leukemia: a report from the Children's Cancer Group. Blood. 2002;99(3):825-833.

27. Conter V, Valsecchi MG, Silvestri D, et al. Pulses of vincristine and dexamethasone in addition to intensive chemotherapy for children with intermediate-risk acute lymphoblastic leukaemia: a multicentre randomised trial. Lancet. 2007;369(9556):123131.

28. De Moerloose B, Suciu S, Bertrand Y, et al. Improved outcome with pulses of vincristine and corticosteroids in continuation therapy of children with average risk acute lymphoblastic leukemia (ALL) and lymphoblastic non-Hodgkin lymphoma (NHL) report of the EORTC randomized phase 3 trial 58951. Blood. 2010;116(1):36-44.

29. Stock W, La M, Sanford B, et al. What determines the outcomes for adolescents and young adults with acute lymphoblastic leukemia treated on cooperative group protocols? A comparison of Children's Cancer Group and Cancer and Leukemia Group B studies. Blood. 2008;112(5):1646-1654. 